\begin{tabular}{|l|l|} 
REVISTA Revista Educación \\
ISSN: 0379-7082 \\
ISSN: 2215-2644 \\
revedu@gmail.com \\
Universidad de Costa Rica \\
Costa Rica
\end{tabular}

\title{
De la entropía social a la entropía educativa. Una reflexión en el contexto colombiano
}

\author{
Cárdenas Messa, Gustavo Adolfo \\ De la entropía social a la entropía educativa. Una reflexión en el contexto colombiano \\ Revista Educación, vol. 44, núm. 1, 2020 \\ Universidad de Costa Rica, Costa Rica \\ Disponible en: http://www.redalyc.org/articulo.oa?id=44060092030 \\ DOI: https://doi.org/10.15517/revedu.v44i1.37100
}

Esta obra está bajo una Licencia Creative Commons Atribución-NoComercial-SinDerivar 3.0 Internacional. 


\section{De la entropía social a la entropía educativa. Una reflexión en el contexto colombiano}

From Social to Educational Entropy. A Reflection on the Colombian Context

Gustavo Adolfo Cárdenas Messa

DOI: https://doi.org/10.15517/revedu.v44i1.37100

Unidad Central del Valle del Cauca, UCEVA, Colombia

Redalyc: http://www.redalyc.org/articulo.oa?id=44060092030

gcardenas@uceva.edu.co

(D) http://orcid.org/0000-0002-7571-9023

Recepción: 27 Mayo 2019

Aprobación: 03 Diciembre 2019

\section{Resumen:}

El presente artículo es una aproximación académica a la migración del concepto de entropía (la segunda ley de la termodinámica) a los fundamentos de la educación. Se basa fundamentalmente en la noción de un mundo cambiante, sometido al azar, a la incertidumbre, que no se ve representado en un sistema educativo estático, con mucha movilidad teórica, pero poca movilidad didáctica y evaluativa. En esta medida, la entropía se convierte en el escenario propicio de las transformaciones que requiere la educación para dar respuestas a las necesidades de una realidad que no es estática. Se busca analizar la educación en el contexto colombiano, como un sistema con bajos niveles de entropía, organizado, sistemático, y poco adaptable a la realidad cambiante de la contemporaneidad.

Palabras Clave: Educación, Entropía, Transformación.

\section{Abstract:}

This study sets forth an academic approach that applies the concept of entropy (the second law of thermodynamics) to the foundations of education. The concept of entropy is based on the notion of uncertainty in a changing world that is subject to chance and which is not represented in a static educational system which possesses a degree of theoretical mobility but has little didactic and evaluative mobility. As a result, entropy becomes a favorable setting for the type of transformation that education must undergo to respond to the needs of a non-static system. This article analyzes the context of the Colombian educational system, a low-entropy system that is organized, systematic and hardly adaptable to the changing actuality of our contemporary world.

KEYWORDS: Education, Entropy, Transformation.

\section{LA ENTROPÍA EN EDUCACIÓN}

La educación es fundamentalmente una acción social y cultural. En esta medida se convierte en un campo intelectual del pensamiento contemporáneo, como objeto de análisis por su impacto en el devenir social. Poner en escena el campo educativo desde sus interacciones con otros modelos teóricos cobra relevancia y vigencia en la medida que estos modelos teóricos aportan a la comprensión del fenómeno educativo en la actualidad. El propósito de esta reflexión, es pues, revisar desde el modelo teórico de las leyes de la termodinámica, particularmente de la segunda ley, un fenómeno educativo el cual tiene que ver con la tendencia de los sistemas, de movilizarse del orden al caos, y eventualmente, la generación de órdenes nuevos.

La educación, la sociología, la psicología, entre otras áreas humanísticas, han traído a su basamento teórico, conceptos que se formaron en otras disciplinas. La resiliencia, por ejemplo, es una traspolación de la ingeniería de materiales a la piscología. La calidad es un aporte de las ingenierías a casi todas las disciplinas, entre ellas las ciencias de la educación. Estos y otros ejemplos muestran ese fenómeno de migración conceptual interdisciplinar que alimentan la comprensión de algunos fenómenos sociales que pueden ser explicados desde las metáforas de las disciplinas naturales.

Uno de esos conceptos está basado en la segunda ley de la termodinámica: La entropía, que se entiende como la tendencia de los sistemas de pasar del orden al caos de manera continua, si no hay manipulación 
del medio en el cual se inscriben esos sistemas. El físico Jeremy England (2015) investigador del M.I.T. lo ejemplifica con una tasa de café caliente. A menos que se manipule el medio ambiente, la tendencia del café es a enfriarse a la medida del medio que lo rodea, es decir, que se pasa de un estado ideal, de orden, a un estado de caos, que en termodinámica tiende al cero respecto al medio ambiente. Otro ejemplo es que hay mayor entropía en el vapor de agua, con sus moléculas dispersas, que, en el estado sólido, con sus moléculas estrechamente unidas

Este concepto se ha visto filtrando hacia algunas reflexiones sobre fenómenos sociales. ¿Una entropía social? ¿Existen fenómenos sociales que tienden al caos, a la entropía? La respuesta no es obviamente sencilla. Como siempre las traspolaciones corren el riesgo de no ser precisas ni responder de forma natural a la teoría que las generó. Ya Diego Linari (2005) ha abordado el concepto en su obra entropía educativa, desde el sistema educativo y desde la perspectiva del sujeto. Igualmente, Sulpicio Vicuña (2018) en su obra La entropia pedagógica, asume la entropía como fenómeno visible en el sistema educativo peruano.

\section{ESCENARIOS EDUCATIVOS}

La educación como fenómeno social tiende a responder a la naturaleza cambiante de las dinámicas sociales. En este sentido se podría pensar en cuatro escenarios fenomenológicos en los cuales se expresa esa respuesta del pensamiento y del hecho educativo:

El primer escenario lo he denominado Cinética del Arraigo, una especie de oxímoron, pero que responde muy bien a la idea: El arraigo genera cierta movilidad que tiene que ver con la defensa de las ideas o de las prácticas a las cuales se está arraigado. Es la energía resultado de esta movilidad, aquella que expresa lo que en la práctica ocurre cuando los docentes presentamos resistencia al cambio. Cualquier alteración de la zona de confort genera incomodidad, muchas veces, incapacidad de adaptarse a tales rupturas. Y generalmente esa resistencia se sustenta en falacias: ;Asi lo he hecho toda la vida y me ha funcionado! ¡Hasta ahora nadie se ha quejado! ¡Pregúntenle a los que fueron mis alumnos desde hace 20 años! ¡Ese cambio no sirve! Y en esa medida, la resistencia argumentada se convierte en una defensa de la razón, o de la verdad concebida desde las propias experiencias.

El segundo escenario es el que he denominado Activismo pedagógico, un mecanismo de defensa interesante, pero que no aporta mucho a las lógicas de la educación. Este responde a las prácticas del cuerpo docente que se quiere adaptar al cambio, y para ello desarrolla una gran cantidad de actividades, ya sea desde lo tecnológico, desde las prácticas didácticas, o desde la concepción discursiva. Pero este sinnúmero de actividades no tiene un norte, no responde a la idea del desarrollo de competencias, o de transversalización de saberes. Son solo hacer, ya sea desde la práctica o desde el discurso. (Esto podría concebirse como un estatismo didáctico con movilidad teórica). Este es un escenario en el cual el personal docente hace mucho, pero aporta poco a la construcción de un proyecto con fines precisos de formación.

El tercer escenario es el de la entropía educativa. Este se presenta tanto en el ámbito administrativo como en la academia. Es un proceso gradual de paso de un estado de desequilibrio con alto grado de entropía a otro con bajo grado de entropía. El paso del caos al orden de forma gradual y sistemática. Ocurre por ejemplo cuando el maestro o la maestra, que ha empezado su vida en las aulas con alegría, ilusiones e ideas, y que ve reflejada en el aula su propia identidad, empieza a perderla con el paso de los años. Esto le lleva a un cumplimiento (cumplo y miento) de su tarea académica, de su horario, de sus cargas cotidianas, sin interés alguno por el aprendizaje. Esto podría equipararse con el síndrome de Burnout ${ }^{[1]}$ (García, 2019); (guardando obviamente las proporciones), el sujeto quemado. Este es un estado que ha sido estudiado como fenómeno psicológico, no solo en los maestros, sino en diferentes profesiones y actividades humanas.

El cuarto escenario que planteo es el transformacionismo pedagógico, la acción educativa asertiva, resultado del establecimiento de unos fines específicos para el acto de educar, consecuencia, (tal vez) de cierta vocacionalidad del maestro y la maestra, que involucra su apasionamiento por la educación. 
Debido a que el primero, segundo y cuarto escenario siempre estarán afectados por la entropía, en la medida que es una manifestación de los sistemas, y en cuanto la educación es un subsistema del entramado social, será este el ámbito de la presente aproximación.

Colombia ha sufrido durante décadas los cambios de políticas públicas que se dan con cada cambio de gobierno. Se podría entender que el sistema educativo está mediado por políticas de gobierno y no de Estado. Un ejemplo concreto es la diferencia de concepción y de programas en los diferentes gobiernos: En el periodo de Gobierno de 2002 a 2010 el plan de desarrollo concibe la educación como "un instrumento para la construcción de la equidad social” (Revista Semana, 2016, pp. 17-19). Es decir, como un medio para el crecimiento económico y de mercado. En el periodo de gobierno 2010 a 2018 se entendió que "la educación es el único camino que a largo plazo nos sacará del subdesarrollo y la pobreza, y que nos permitirá lograr la prosperidad" (Revista Semana, 2016, pp. 17-19), estas dos percepciones determinaros dos estilos de política educativa, el primero que privilegió al Servicio Nacional de Aprendizaje (SENA) como institución de formación técnica laboral líder, mientras que el segundo privilegió el sistema educativo formal, en busca de propiciar mayores oportunidades de desarrollo. En ambos casos se presenta una perspectiva de nación diferente a partir de sus fines.

\section{HaCIA UNA COMPRENSIÓN DE LA ENTROPÍA EDUCATIVA}

La entropía, desde la perspectiva de la termodinámica, se puede entender de forma positiva, cuando esta se incrementa y aumenta la desorganización molecular, haciendo que el azar juegue un papel más importante en el logro del equilibrio. Y de forma negativa, cuando hay mayor orden, es decir, menor entropía. Desde la perspectiva de la educación, la entropía puede ser positiva o negativa en la media en que responde a diferentes escenarios culturales o situacionales.

Una entropía positiva (desorden creciente en el sistema) muchas veces puede ser un mecanismo de reorganización (Cazau, 1995), o puede ser síntoma de un sistema desarticulado, sin una hoja de ruta, sin una finalidad, o con finalidades disímiles y dispersas.

La negentropía (entendida como entropía negativa), es para el sistema educativo una tendencia a la organización, a la coherencia, o a la minimización del azar (cosa bastante improbable en procesos culturales). Es la eterna distinción entre el ser y el deber ser.

La educación debe responder a las condiciones cambiantes del entorno, adaptando sus prácticas y sus contenidos a las necesidades históricas (actuales) del estudiantado, y a sus intereses de formación. En esta medida la educación está sometida a altos niveles de entropía, pues hay una gran dependencia de las condiciones cambiantes del entorno. Sin embargo, la realidad muestra que las prácticas educativas (con notables excepciones) son altamente tradicionales, organizadas, con bajos niveles de entropía, y con tendencia a mantener el statu quo. Esta negentropía si bien permite un cierto control (un poco ilusorio) del proceso educativo, no permite que se introduzcan al sistema, nuevas formas de comprensión del mundo y de construcción de sentidos.

Los modelos teóricos han evolucionado mucho en educación. Las actuales reflexiones sobre las pedagogías activas (Ausubel, 1983; Berstein, 1988; Garner, 1987), y las más contemporáneas reflexiones sobre pedagogías sociales (Bauman, 2000; De Sousa, 2012; Quintar, 1998), tienden a comprender la realidad social como un sistema o un conjunto de sistemas altamente cambiantes, a los cuales la educación debe dar respuesta como subsistema del macro sistema cultural. Esto implica que la educación es (o debe ser) un sistema de altos niveles de entropía, capaz de reorganizarse y resignificarse permanentemente.

Si concebimos los diferentes niveles de formación, podríamos decir que en educación inicial, hay mayor claridad sobre cuál es la finalidad primaria de esta etapa (la socialización, el desarrollo de interacciones con el mundo, la motricidad, el descubrimiento de su realidad circundante), sin embargo aún se desarrollan 
actividades puramente académicas, es decir, de enseñanza de contenidos, donde la prioridad no es el desarrollo de competencias básicas de comunicación e interacción, sino el aprendizaje de datos o de habilidades.

En educación básica y secundaria, la preocupación ha pasado de los contenidos a los resultados de las pruebas de estado, y esto, si bien apoya la idea del desarrollo de competencias, no involucra de manera activa al estudiantado. La finalidad ha cambiado, pero los modelos que buscan esa finalidad son los mismos.

En educación terciaria (los diferentes niveles de educación superior) queda la tarea de ser remedial de las deficiencias de los niveles previos, o cumplir con su misión profesionalizante. Generalmente, el intento de armonizar las necesidades del alumnado con el compromiso de formación termina en un híbrido que no satisface ni un lado ni el otro.

Esta manifestación de negentropía tiende a mantener cierto orden, cumplir de manera exhaustiva con las exigencias y directrices ministeriales (Estándares, lineamientos, DBA -Derechos Básicos de Aprendizaje-, currículo sugerido, mallas de aprendizaje), mejorar los puntajes obtenidos en las pruebas Saber, atención a los proyectos institucionales, entre otras fundamentaciones bastante estáticas. En este escenario, lograr que el proceso educativo se transforme en la medida que la teoría y la realidad se transforman, es poco probable.

Esta es la existencia de muchas instituciones públicas en Colombia, donde el cumplimiento de exigencias se ha convertido en un obstáculo, antes que en una oportunidad de mejora de la calidad educativa (Ver Mineducación, 2019).

\section{INSTITUCIONES EDUCATIVAS Y ENTROPÍA}

Otro aspecto importante se encuentra relacionado con la naturaleza de las instituciones. Mientras que las I.E. del Estado no desarrollan (en su mayoría) una línea pedagógica orientadora, sino que se mantienen en el marco ministerial, las I.E. privadas buscan establecer elementos diferenciadores que potencien su presencia en el mercado educativo. Es por esto que la lucha por los mejores puntajes en las pruebas de estado, se dan entre las instituciones privadas; mientras que las públicas hacen la tarea de mantenerse en la media nacional, en el mejor de los casos. Esto se puede evidenciar con los resultados de las pruebas saber 11 del segundo semestre de 2019, tanto a nivel nacional, como por regiones.

Los sistemas abiertos, aquellos que más se permean de las realidades exteriores, tienden igualmente a una mayor entropía que aquellos que están más cerrados, o más aislados en lo ideológico, geográfico o educativo. Un sistema educativo cerrado o aislado, (ya sea porque el profesorado no quiere incorporar cambios en su práctica, o porque no hay acceso a recursos por distancia geográfica, o porque su dirección no permite riesgos en los procesos de formación), tiende a manifestar negentropía, o entropía negativa, esto es, un mayor orden, pero menor capacidad de evolución y de adaptación.

Esta entropía negativa puede presentarse desde la perspectiva del cuerpo docente, como lo referencia Jacques Ranciere (2003) en la medida que este no se forme, no se apropie de nuevas perspectivas teóricas, no incorpore el uso de nuevas tecnologías a su praxis, o desarrolle cierto activismo pedagógico que no deriva en una intencionalidad pedagógica clara. Desde la perspectiva de las instituciones, en la medida en que no fomentan la investigación, no propician espacios de reflexión pedagógica ni la incorporación de modelos activos o sociales que se acerquen más a la realidad del contexto.

Bordieu (1984) planteaba en su teoría, que los habitus se definen a partir de las prácticas sociales repetitivas, social o individualmente incorporadas, y que responden a lo concebido como adecuado, correcto o tradicionalmente validado. Se puede inferir aquí una especie de resistencia paradigmática sobre la cual ya nos había advertido Kuhn (1962), en su Estructura de las revoluciones cientificas.

Es claro pues, que a mayor entropía, mayores posibilidades de emancipación intelectual (Ranciere, 2003), mayores posibilidades de constitución de capitales sociales de contexto (Bordieu, 1984), y mayor posibilidad de incorporación de modelos educativos propios de las dinámicas contemporáneas de los sujetos (Garner, 1987). En su opuesto: A menor entropía, mayor organización estandarizada, menor capacidad de adaptación 
a los cambios, mayor riesgo de pérdida de utilidad práctica del sistema educativo formal y menor capacidad de respuesta a los cambiantes problemas de la sociedad contemporánea (Bauman, 2000).

Actualmente existe una tendencia fuerte a nivel de políticas estatales y multinacionales, en relación con la educación; prueba de ello es la naturaleza de las pruebas de estado Saber, y las pruebas internacionales como PISA o PIRLS. Si bien la búsqueda de desarrollo de competencias es una finalidad educativa loable, existe una obviedad relacionada con la heterogeneidad de los contextos, y la precariedad de las pruebas para dar respuesta a las necesidades de formación en esos contextos.

Estandarizar siempre ha sido la solución económica y técnicamente viable, que, sin embargo, no garantiza respuestas a las preguntas específicas de las regiones, ni soluciones a las problemáticas propias de cada escenario educativo. En este caso los criterios de evaluación van completamente en contravía del pensamiento pedagógico contemporáneo, que nos dice, desde Estela Quintar (1998), por ejemplo, que la verdadera pedagogía es la de la potenciación de las capacidades del sujeto, o Amartya Sen (1999) que nos recuerda que el desarrollo colectivo es posible en la medida que se propicie individualmente. O incluso Manfred Max Neef (1993), quien nos dice que el desarrollo debe ser a escala humana, reconociendo las particularidades económicas, sociales culturales y cognitivas de cada región y de cada sujeto. Esto pasa a través de lo que De Sousa (2012) llama, la Ecología de los saberes, esa diversidad propia de la escena humana en la ciencia, la técnica y las humanidades, todas enmarcadas en sus propias realidades crono-geográficas, o históricogeográficas si se prefiere.

Esta contradicción en la cual la teoría cambia mucho más rápidamente que la práctica, y mucho más que las normas que tipifican la práctica, evidencian de forma alarmante la brecha que hay entre la educación y la realidad.

\section{Los REZAGos HISTÓRICOS DE LA EDUCACIÓN EN EL ÁMBITO COLOMBIANO.}

Esta realidad puede ser extrapolada a muchos otros ámbitos nacionales, y para naturalizar este ejercicio, es necesario reconocer que de esta forma la educación nacional se ha venido quedando rezagada frente a los retos y desafíos del mundo poscontemporáneo (aunque esta forma de taxonomía sea arbitraria), o que, en el mejor de los casos, la realidad social rebasó la realidad educativa de una forma alarmante.

La profunda revolución que ha representado la interconexión tecnológica del mundo, y la forma como esto ha afectado nuestra manera de relacionarnos, de aprender y de construir sentidos; las implicaciones profundas en la cultura en relación con los desarrollos tecnológicos en salud, comunicaciones, seguridad, servicios sociales (entre muchos otros), no pueden ser desconocidas por la educación. Este distanciamiento no es otra cosa que un detenimiento, una forma de status quo, que implica en sí mismo un retroceso, si el punto de referencia cambia constantemente. Y sí cambia.

La frase lapidaria de Fernando Figueira (2008), nos dice que somos maestros del siglo XX, formando personas del siglo XXI con modelos metodológicos y contenidos del siglo XIX, lo cual, por sí mismo, valdría para alarmarnos y ponernos, no a la defensiva, sino a la ofensiva; no contra el sistema, o contra las normas, sino contra nuestras propias incapacidades frente al devenir social.

Un sistema educativo propicio a la entropía, capaz de asumir la desestabilización de la cultura, el azar, la incertidumbre de la cual nos habla Edgar Morín (1999), y capaz de ser realmente agente de cambio, punta de lanza en las transformaciones sociales, obliga también a que el maestro y maestra no sean observadores pasivos, sino actores comprometidos, qué superando las ambigüedades y estatismos miopes de los dirigentes de turno, puedan, desde su propia práctica, ser agentes de esta dinámica social. Esto se ve necesariamente reflejado en el estudiante y sus dinámicas:

...el individuo alcanza un equilibrio (precario, frágil, inestable), que no le exenta de devenir en desorden y degradación del modo que lo sugiere el principio de la entropía, debido a la estabilidad que se alcanza (que reduce incertidumbre) y que incluso 
es estimulada con evaluaciones positivas: elegir las respuestas correctas, no equivocarse para no ser reprobado (Arriaga, 2017, p. 35).

Para concluir, recurro a los escenarios que enuncié al comienzo, en los cuales, el transformacionismo pedagógico (entendido como la movilización de los saberes desde el ejercicio didáctico, que involucra, no solo el elemento cognitivo, sino el elemento axiológico y social) no implica anarquía, no implica volubilidad, o incapacidad de reorganización a partir de nuevos órdenes, sino que implica capacidad de adaptación, es decir, la capacidad de reconocer los cambios, aceptar las nuevas condiciones, y hacer modificaciones significativas a las prácticas educativas; y más aún, capacidad para ser los generadores de cambio, para ser parte de la vanguardia que propicia las transformaciones en una sociedad, cuya identidad cada vez se ve amenazada por crecimientos desordenados y sin soportes éticos que los sustenten.

Las transformaciones en el sistema educativo que hoy demanda la sociedad, son, mucho más que cambios en los contenidos, para adaptarlos a los nuevos saberes: Implican la transformación de las percepciones que las maestras y los maestros tenemos del estudiantado, de la realidad y de nuestras propias prácticas educativas.

Se puede inferir que el sistema educativo colombiano está sometido a un elevado nivel de entropía, en la medida que es vulnerable a los cambios permanentes en la legislación educativa, asociada a los conceptos que sobre ello tienen los diferentes gobiernos, los cuales no permiten convertir la educación en una política de estado, consistente, coherente, congruente y contextual. Igualmente, que esta entropía se ve manifestada en la gestión pedagógica del docente, derivada, entre otros aspectos, de las carencias motivacionales que propician mejoramientos continuos, resignificación de la educación a partir de las necesidades de los contextos, y de las nuevas formas de pensamiento pedagógico.

\section{ReFERENCIAS}

Arriaga, J. L. (2017). Sistema, autopoiesis y entropía en los desafíos educativos contemporáneos. Educación y Humanismo, 19 (33), 271-288. doi: http://dx.doi.org/10.17081/eduhum.19.33.2644

Ausubel, D. (1983). Psicología educativa: Un punto de vista cognitivo. México: Trillas.

Bauman, S. (2000). Modernidad líquida. Buenos Aires: Fondo de Cultura Económica.

Berstein, B. (1988). Poder, educación y conciencia. Santiago: CIDE.

Bordieu, P. (1984). Sociología y cultura. Argentina: Grijalbo.

Cazau, P. (1995). La teoría del caos. Recuperado de http://www.uca.edu.sv/facultad/chn/c1170/Teoria\%20del\%20 caos.pdf

De Sousa, S. B. (2012). Epistemologias del sur. México: Siglo XXI Editores.

England, J. (2015). Entropia social y teoría de caos social. Recuperado de https://www.englandlab.com/publications. html

Figueira, F. (2008). La desigualdad como clave social del desarrollo maniatado. Buenos Aires: Clacso.

García, J. (2019). Burnout (sindrome del quemado). Recuperado de https://psicologiaymente.com/organizaciones/b urnout-sindrome-del-quemado

Gardner, H. (1987). Las estructuras de la mente. México: Fondo de Cultura Económica.

Kuhn, T. (1962). Teoría de las revoluciones cientificas. México: Fondo de Cultura Económica.

Linari, D. (2005). Entropia Educativa. Buenos Aires: Dunken.

Max, N. M. (1993). Desarrollo a escala bumana. Icaria: Barcelona.

Mineducación. (2019). Ministerio de Educación Nacional. Recuperado de https://www.mineducacion.gov.co/portal /Preescolar-basica-y-media/

Morín, E. (1999). Los siete saberes necesarios para la educación del futuro. Paris: UNESCO.

Quintar, E. (1998). La enseñanza como puente a la vida. México: IPECAL. 
Ranciere, J. (2003). El maestro ignorante. Barcelona: Laertes.

Revista Semana. (2016). La educación de Uribe Vrs la de Santos. Recuperado de https://bit.ly/35K4Acb Sen, A. (1999). Desarrollo y libertad. Barcelona: Planeta.

Vicuña, S. (2018). La entropía pedagógica. Lima: Yopublico.

\section{Notas}

[1] Síndrome del sujeto quemado.

\section{BY-NC-ND}

